\title{
SARS-CoV-2 Infection with Associated Rhabdomyolysis and Probable Myocarditis
}

\author{
Franco Murillo ${ }^{1}$, Gastón Ramos ${ }^{1}$, José Luis del Pozo ${ }^{2}$, Luis Manuel Valdez ${ }^{1,3,4}$, Jorge Zagaceta ${ }^{1,3}$ \\ ${ }^{1}$ Clínica Anglo-Americana, Lima, Peru \\ ${ }^{2}$ Clínica Universidad de Navarra, Pamplona, Spain \\ ${ }^{3}$ Facultad de Medicina Humana, Universidad de Piura, Lima, Peru \\ ${ }^{4}$ Departamento de Medicina, Universidad Peruana Cayetano Heredia, Lima, Peru
}

\section{Doi: 10.12890/2020_001867 - European Journal of Case Reports in Internal Medicine - ๔ EFIM 2020}

Received: 03/07/2020

Accepted: $13 / 07 / 2020$

Published: $19 / 08 / 2020$

How to cite this article: Murillo F, Ramos G, del Pozo JL, Valdez LM, Zagaceta J. SARS-CoV-2 infection with associated rhabdomyolysis and probable myocarditis. EJCRIM 2020;7: doi:10.12890/2020_001867.

Conflicts of Interests: The Authors declare that there are no competing interests.

This article is licensed under a Commons Attribution Non-Commercial 4.0 License

\section{ABSTRACT}

We describe a patient with SARS-CoV-2 and severe pneumonia who required mechanical ventilation and developed associated rhabdomyolysis with probable myocardial involvement as evidenced by cardiac enzyme abnormalities and echocardiographic findings. Repeat testing should be done in cases highly suspicious for SARS-CoV-2 as initial molecular tests may be negative, as in our case.

\section{LEARNING POINTS}

- SARS-CoV-2 infection may be associated with rhabdomyolysis and myocarditis.

- Negative results for SARS-CoV-2 despite a clinical presentation suggestive of COVID-19 disease should be treated with caution.

- Drugs known to cause rhabdomyolysis and myocarditis should be carefully reviewed when treating SARS-CoV-2 patients.

\section{KEYWORDS}

SARS-CoV-2, rhabdomyolysis, myocarditis

\section{INTRODUCTION}

On 11 March 2020, the World Health Organization (WHO) declared that the outbreak of severe acute respiratory syndrome (COVID-19) caused by the novel coronavirus SARS-CoV-2 was a pandemic ${ }^{[1]}$. As of 3 July 2020 there have been more than 10 million infections with thousands of deaths worldwide. The first report on COVID-19 was published by Huang et al. ${ }^{[2]}$. Further study has shown that SARS-CoV-2 uses the widely expressed angiotensin-converting enzyme 2 (ACE2) receptor to obtain entry to cells ${ }^{[3]}$.

While some patients are asymptomatic, others develop pneumonia and acute respiratory distress syndrome (ARDS) ${ }^{[4]}$. The most frequent symptoms are cough, fever, malaise, myalgia and diarrhoea, while bilateral ground-glass opacifications are the most common chest CT finding ${ }^{[2]}$. Sepsis, respiratory failure and ARDS are frequent complications ${ }^{[5]}$. Rhabdomyolysis and myocarditis have been described as potential late complications of SARS-CoV-2 infection. Early identification and treatment are critical to decrease mortality. To the best of our knowledge, we describe the first case of associated rhabdomyolysis and probable myocarditis in a patient with SARS-CoV-2 infection.

\section{CASE DESCRIPTION}

A 48-year-old man arrived in Peru from Spain with no symptoms 10 days before hospital admission. Three days after arrival in the country, he developed general malaise, asthenia, myalgias, dry cough, progressive dyspnoea and fever. Two days later he self-medicated with amoxicillin plus clavulanic acid without improvement. Three days later, an ambulatory nasopharyngeal swab was taken for SARS-CoV-2 
real-time polymerase chain reaction testing due to clinical worsening. The following days he continued to deteriorate and was admitted to our institution. At that time a second nasopharyngeal swab for SARS-CoV-2 RT-PCR testing was taken. Both tests were reported later as negative.

On admission, the patient's temperature was $38.8^{\circ} \mathrm{C}$, his blood pressure was $130 / 70 \mathrm{mmHg}$, his pulse was 93 beats per minute, his respiratory rate was 30 breaths per minute and oxygen saturation was $95 \%$ on room air. Use of accessory muscles, slurred speech and a poor ventilatory pattern were noted. On chest auscultation, a bilateral decrease in breath sounds was identified. The rest of the examination was non-contributory except for a BMI of $31 \mathrm{~kg} / \mathrm{m}^{2}$. Initial laboratory work-up showed hypoxaemia, mildly elevated creatinine phosphokinase (CPK), lactic dehydrogenase, C-reactive protein and fibrinogen, and slightly elevated liver enzymes (Table 1). The chest CT showed bilateral ground-glass opacifications (Fig. 1A).

Treatment was started according to our current COVID-19 protocol with ceftriaxone, azithromycin, oseltamivir and hydroxychloroquine along with standard supportive treatment. During hospitalization day 1 , respiratory insufficiency worsened rapidly with a $\mathrm{PaO}_{2} / \mathrm{FiO}{ }_{2}$ of $<200$. The patient was transferred to the intensive care unit (ICU) where he was intubated and placed on mechanical ventilation.

Due to clinical worsening, high PEEP levels were required as well as pronation. A more comprehensive laboratory panel was requested, along with a third sample for SARS-CoV-2 RT-PCR testing from tracheal aspiration. The respiratory FilmArray panel and admission blood cultures were negative. On hospitalization day 4, CPK values started to rise until hospitalization day 7, reaching a value of 10,768 U/I; pigmenturia was also noted (Fig. 1B). Aggressive fluid therapy was initiated. Renal function was not compromised. As the CPK values began to decline, troponin T values increased up to $0.168 \mathrm{ng} / \mathrm{ml}$ (Fig. 1B and Table 2). The patient developed new onset hypertension (up to $160 / 100$ $\mathrm{mmHg}$ ) and atrial fibrillation. An echocardiogram was performed revealing moderate dilatation of the right chambers, apical dyskinesis of the right ventricle and mild pericardial effusion. The ejection fraction was normal.

A.
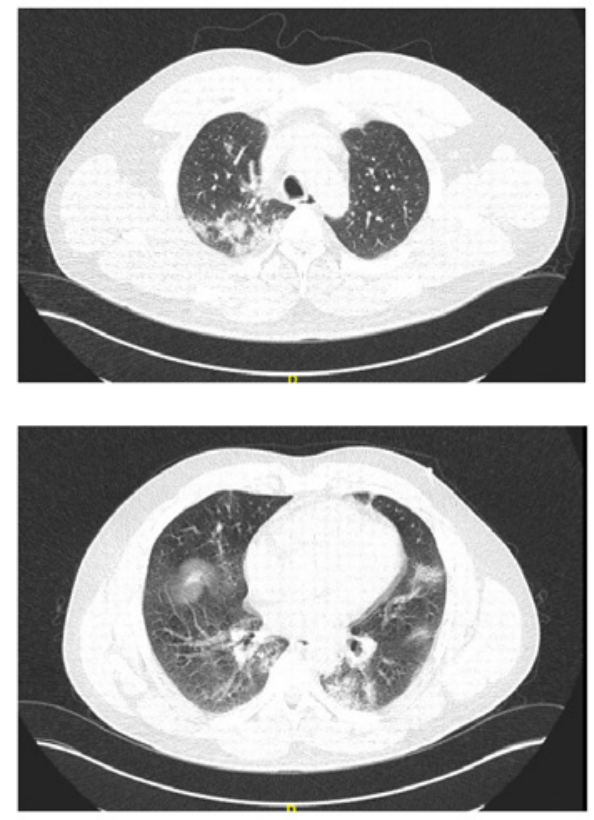

B.

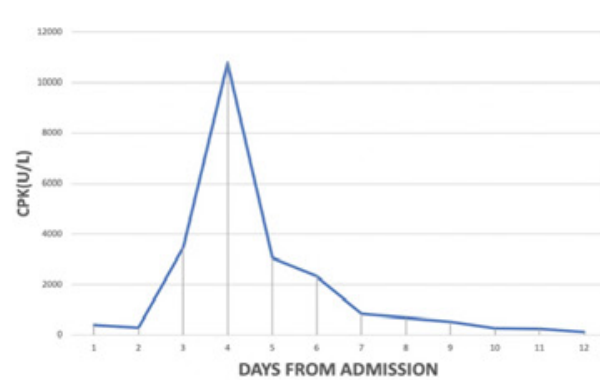

DAYS FROM ADMISSION

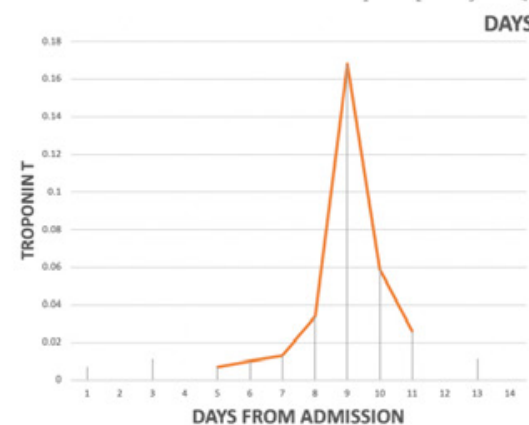

Figure 1 (A) The chest CT scan on admission shows bilateral ground-glass opacifications. (B) Daily progression for rhabdomyolysis and myocarditis serological markers since admission, showing a clear peak and return to normal values a short time later

\section{DISCUSSION}

Rhabdomyolysis is a potentially fatal disease defined by elevated CPK levels of up to 10 times normal, followed by a rapid decrease ${ }^{[6]}$. Aetiologies include viral infections, such as influenza, coxsackievirus and Epstein-Barr virus. In addition, during the previous outbreak of SARS-CoV-1, some cases of rhabdomyolysis were reported ${ }^{[7]}$. To the best of our knowledge, we present the first case of severe SARS-CoV-2 infection associated with rhabdomyolysis and probable myocarditis as a late complication. 


\begin{tabular}{|c|c|c|}
\hline General & Admission 20/03 & Reference range \\
\hline Haemoglobin (g/dl) & 15,8 & $13.6-17.5$ \\
\hline Platelet count (per mm ${ }^{3}$ ) & 251.000 & $139,000-403,000$ \\
\hline White cell count (per mm³) & 7.550 & $4,400-11,300$ \\
\hline Absolute lymphocyte count (per $\mathrm{mm}^{3}$ ) & 1.661 & $1,000-4,000$ \\
\hline Procalcitonin (ng/ml) & 0,07 & $<0.50$ \\
\hline C-Reactive protein (mg/dl) & 7,03 & $0.00-0.50$ \\
\hline Creatinine (mg/dl) & 1,07 & $0.5-1.20$ \\
\hline Urea $(\mathrm{mg} / \mathrm{dl})$ & 17 & $11.0-50$ \\
\hline D-Dimer $(\mu \mathrm{g} / \mathrm{ml})$ & 0,2 & $11.0-0.50$ \\
\hline $\mathrm{LDH}(\mathrm{U} / \mathrm{I})$ & 401 & $135-225$ \\
\hline AST (U/I) & 65 & $0.0-40$ \\
\hline ALT (U/I) & 56 & $0.0-38$ \\
\hline Sodium (mEq/l) & 135,7 & $135-145$ \\
\hline Potassium (mEq/l) & 4,08 & $3.5-5.5$ \\
\hline Chloride(mEq/l) & 96,7 & $22-34$ \\
\hline Glucose (mg/dl) & 106 & $70-100$ \\
\hline \multicolumn{3}{|l|}{ Arterial blood gases } \\
\hline $\mathrm{pH}$ & 7,44 & $7.35-7.43$ \\
\hline $\mathrm{PCO}_{2}$ & 39 & $32-43$ \\
\hline $\mathrm{PO}_{2}$ & 57 & $80-100$ \\
\hline $\mathrm{HCO}_{3}$ & 26,5 & $22-28$ \\
\hline Immunological panel & Value & Reference range \\
\hline ESR (mm/hour) & 82 & $0-15$ \\
\hline C3 (mg/dl) & 140,9 & $90-180$ \\
\hline $\mathrm{C} 4(\mathrm{mg} / \mathrm{dl})$ & 20,1 & $10.0-40$ \\
\hline Haptoglobin (mg/dl) & 465,08 & $30-200$ \\
\hline C-ANCA $(\mathrm{U} / \mathrm{ml})$ & 0,1 & $<5.0$ \\
\hline P-ANCA (U/ml) & 0,2 & $<5.0$ \\
\hline Anti-DNA & Negative & - \\
\hline Anti-Nucelar Ab & $1 / 80$ & - \\
\hline Anti-GBM & Negative & - \\
\hline
\end{tabular}

Table 1. Admission laboratory and immunological panel results

\begin{tabular}{|l|l|l|l|l|l|l|l|l|l|l|l|l|l|l|l|}
\hline $\begin{array}{l}\text { Rhabdomyolysis } \\
\text { and myocarditis } \\
\text { markers }\end{array}$ & $20 / 03$ & $22 / 03$ & $24 / 03$ & $25 / 03$ & $26 / 03$ & $27 / 03$ & $29 / 03$ & $30 / 03$ & $01 / 04$ & $03 / 04$ & $4 / 04$ & $6 / 04$ & $12 / 04$ & $14 / 04$ & $\begin{array}{l}\text { Reference } \\
\text { range }\end{array}$ \\
\hline CPK (U/l) & 405 & 296 & 3.469 & & & 10.768 & 3.041 & 2.335 & 839 & 689 & 522 & 256 & 250 & 121 & $26-190$ \\
\hline CPK-MB (ng/ml) & 1,09 & 1,13 & 13,36 & & 30,3 & 26,52 & 4,17 & & & 6,7 & 3,28 & & 2,14 & 1,99 & $<5.0$ \\
\hline Troponin-T & 0,007 & & 0,011 & & 0,007 & 0,01 & 0,013 & 0,034 & 0,168 & 0,059 & 0,026 & & 0,011 & & $\begin{array}{l}0.000- \\
0.014\end{array}$ \\
\hline Pro-BNP $(\mathrm{pg} / \mathrm{ml})$ & & & 304,2 & 199,8 & 337,9 & & & & 85,75 & 63,6 & & & & & $0.00-125$ \\
\hline
\end{tabular}

Table 2. Evolution of rhabdomyolysis and myocarditis markers

Our patient met the criteria for rhabdomyolysis as his CPK values increased to over 10,000 U/I on hospitalization day 7 and progressively decreased to near normal values 3 days later. The most common clinical features of rhabdomyolysis are myalgias, pigmenturia and muscle weakness ${ }^{[6]}$. Although our patient had myalgia at initial presentation, at that time the CPK values were only mildly elevated. However, these initial values and myalgia could also be explained by early SARS-CoV-2 infection as previously described ${ }^{[8]}$. By the time the CPK values had dramatically increased, the only clinical feature of rhabdomyolysis was pigmenturia. 
It should be noted that we immediately reviewed and withdrew all potential drugs that could worsen rhabdomyolysis and started aggressive fluid therapy. Myalgias are one of the most common symptoms of SARS-CoV-2 infection. Therefore, early identification of overlapping rhabdomyolysis may be neglected in the early stages of SARS-CoV-2 infection.

During the 2003 SARS-CoV-1 outbreak, many reports of associated rhabdomyolysis were published, with most patients developing acute kidney injury and requiring haemodialysis, resulting in high mortality ${ }^{[7]}$. However, our patient did not progress to acute kidney injury due to prompt identification and early fluid therapy. Further research on the incidence and mortality of SARS-CoV-2-associated rhabdomyolysis is needed. Meanwhile, early identification and treatment are the cornerstones of management.

Although rhabdomyolysis has a diverse aetiology, the underlying pathological processes lead to a common end: the depletion of cellular energy, rupture of the plasma membrane and a subsequent increase in intracellular calcium leading to cell death ${ }^{[6]}$. It has been postulated that rhabdomyolysis secondary to an infectious aetiology may be due to direct damage by the pathogen or to an exaggerated inflammatory response ${ }^{[6]}$. Similar theories have been proposed for SARS-CoV-1 and SARS-CoV-2-associated rhabdomyolysis ${ }^{[7]}$. However, there have been no reports of virus found in muscle tissue or cytopathic damage ${ }^{[9]}$. This favours the theory of rhabdomyolysis being caused by the characteristic exaggerated inflammatory response of SARS-CoV-2 infection ${ }^{[10]}$. Because we did not have a muscle biopsy specimen, we were unable to rule out direct viral damage of muscle tissue in our patient. Nevertheless, we suggest that the damage was caused by an exaggerated inflammatory response, due to the patient's highly elevated inflammatory markers, such as C-reactive protein, ferritin and haptoglobin, by the time of rhabdomyolysis diagnosis.

Myocarditis is defined as inflammation of the myocardium diagnosed by established histological, immunological and immunohistochemical criteria ${ }^{[11]}$. However, only some patients with clinically defined myocarditis undergo a biopsy, so others are diagnosed based on symptoms, and laboratory and imaging studies, which include chest pain, shortness of breath, atrial fibrillation, elevated troponin values, and electrocardiogram and echocardiogram alterations ${ }^{[12]}$. Our patient showed some of those features, including elevated troponin levels and an abnormal echocardiogram.

Myocarditis can also be caused by a wide range of viral infections, including SARS-CoV-2 ${ }^{[11]}$. The mechanism of myocardial injury due to SARS-CoV-2 is still under investigation, but the presence of ACE2 receptors in heart tissue could definitely cause direct viral damage ${ }^{[3]}$. Prompt identification of myocarditis in SARS-CoV-2 patient is critical in order to reduce mortality.

Our report has some limitations, such as the lack of muscle and endomyocardial biopsy and the concomitant use of drugs known to cause rhabdomyolysis at the time of diagnosis.

Finally, we think it is important to consider repeat testing for the diagnosis of SARS-CoV-2 infection in cases with a high index of suspicion. Our patient had two initial negative tests, days apart. The positive sample was obtained from a tracheal aspirate after intubation.

\section{REFERENCES}

1. Velavan TP, Meyer CG. The COVID-19 epidemic. Trop Med Int Health 2020;25:278-280.

2. Huang C, Wang Y, Li X, Ren L, Zhao J, Hu Y, et al. Clinical features of patients infected with 2019 novel coronavirus in Wuhan, China. Lancet 2020;395:497-506.

3. Wang Q, Zhang Y, Wu L, Niu S, Song C, Zhang Z, et al. Structural and functional basis of SARS-CoV-2 entry by using human ACE2. Cell 2020;181(4):894-904.e9.

4. Lai C-C, Liu YH, Wang C-Y, Wang Y-H, Hsueh S-C, Yen M-Y, et al. Asymptomatic carrier state, acute respiratory disease, and pneumonia due to severe acute respiratory syndrome coronavirus 2 (SARS-CoV-2): facts and myths. J Microbiol Immunol Infect 2020;53(3):404-412.

5. Zhou F, Yu T, Du R, Fan G, Liu Y, Liu Z, et al. Clinical course and risk factors for mortality of adult inpatients with COVID-19 in Wuhan, China: a retrospective cohort study. Lancet 2020;395:1054-1062.

6. Zutt R, van der Kooi AJ, Linthorst GE, Wanders RJA, de Visser M. Rhabdomyolysis: review of the literature. Neuromuscul Disord 2014;24:651-659.

7. Wu V-C, Hsueh P-R, Lin W-C, Huang J-W, Tsai H-B, Chen Y-M, et al. Acute renal failure in SARS patients: more than rhabdomyolysis. Nephrol Dial Transplant 2004;19:31803182.

8. Feng Y, Ling Y, Bai T, Xie Y, Huang J, Li J, et al. COVID-19 with different severity: a multi-center study of clinical features. Am J Respir Crit Care Med 2020;201(11):1380-1388.

9. Hsiao C-H, Chang M-F, Hsueh P-R, Su I-J. Immunohistochemical study of severe acute respiratory syndrome-associated coronavirus in tissue sections of patients. J Formos Med Assoc 2005;104:150-156.

10. Hamel Y, Mamoune A, Mauvais F-X, Habarou F, Lallement L, Romero NB, et al. Acute rhabdomyolysis and inflammation. J Inherit Metab Dis 2015;38:621-628.

11. Pollack A, Kontorovich AR, Fuster V, Dec GW. Viral myocarditis--diagnosis, treatment options, and current controversies. Nat Rev Cardiol 2015;12:670-680.

12. Fung G, Luo H, Qiu Y, Yang D, McManus B. Myocarditis. Circ Res 2016;118:496-514. 